\title{
Analysis of microRNAs, phased small interfering RNAs and their potential targets in Rosa rugosa Thunb.
}

Junqiang Guo ${ }^{1 \dagger}$, Qingyi Wang ${ }^{1 \dagger}$, Li Liu², Shuchao Ren², Shipeng Li², Peiran Liao², Zhigang Zhao ${ }^{3}$, Chenyu Lu $\mathrm{Lu}^{3}$, Bingbing Jiang ${ }^{3}$, Ramanjulu Sunkar ${ }^{4}$ and Yun Zheng ${ }^{1,3^{*}}$ (D)

From 17th International Conference on Bioinformatics (InCoB 2018): Genomics

New Delhi, India. 26-28 September, 2018

\section{Abstract}

Background: MicroRNAs (miRNAs) are small non-coding RNAs that play important roles by regulating other genes. Rosa rugosa Thunb. is an important ornamental and edible plant, yet there are only a few studies on the miRNAs and their functions in R. rugosa.

Results: We sequenced 10 samll RNA profiles from the roots, petals, pollens, stamens, and leaves and 4 RNA-seq profiles in leaves and petals to analysis miRNA, phasiRNAs and mRNAs in R. rugosa. In addition, we acquired a degradome sequencing profile from leaf of $R$. rugosa to identify miRNA and phasiRNA targets using the SeqTar algorithm. We have identified 321 conserved miRNA homologs including primary transcripts for 25 conserved miRNAs, and 22 novel miRNAs. We identified 592 putative targets of the conserved miRNAs or tasiRNAs that showed significant accumulations of degradome reads. We found differential expression patterns of conserved miRNAs in five different tissues of R. rugosa. We identified three hundred and thirty nine 21 nucleotide (nt) PHAS loci, and forty nine 24 nt PHAS loci, respectively. Our results suggest that miR482 triggers generations of phasiRNAs by targeting nucleotide-binding, leucine-rich repeat (NB-LRR) disease resistance genes in $R$. rugosa. Our results also suggest that the deregulated genes in leaves and petals are significantly enriched in GO terms and KEGG pathways related to metabolic processes and photosynthesis.
\end{abstract}

Conclusions: These results significantly enhanced our knowledge of the miRNAs and phasiRNAs, as well as their potential functions, in R. rugosa.

Keywords: Rosa rugosa Thunb., High-throughput sequencing, microRNA, Phased small interfering RNA (phasiRNA), SeqTar, Degradome, Bioinformatics

\footnotetext{
*Correspondence: zhengyun5488@gmail.com

† Junqiang Guo and Qingyi Wang contributed equally to this work.

${ }^{1}$ Faculty of Information Engineering and Automation, Kunming University of

Science and Technology, Kunming 650500, China

${ }^{3}$ Yunnan Key Laboratory of Primate Biomedical Research, Institute of Primate

Translational Medicine, Kunming University of Science and Technology,

Kunming 650500, China

Full list of author information is available at the end of the article
}

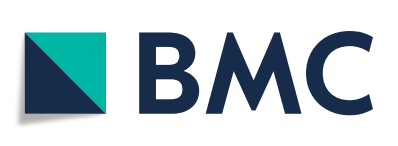

(c) The Author(s). 2019 Open Access This article is distributed under the terms of the Creative Commons Attribution 4.0 International License (http://creativecommons.org/licenses/by/4.0/), which permits unrestricted use, distribution, and reproduction in any medium, provided you give appropriate credit to the original author(s) and the source, provide a link to the Creative Commons license, and indicate if changes were made. The Creative Commons Public Domain Dedication waiver (http://creativecommons.org/publicdomain/zero/1.0/) applies to the data made available in this article, unless otherwise stated. 


\section{Background}

Small non-coding RNAs (ncRNAs), ranging in sizes between 20 and 30 nucleotides, play crucial roles in the biological processes of almost all living organisms. MicroRNAs (miRNAs) and endogenous small interfering RNAs (siRNAs) are two types of small ncRNAs [1]. Under the action of RNA polymerase II, the MIR gene is transcribed into a primary transcript called pri-miRNA [2]. The plant pri-miRNA of typical hairpin-like structure is cleaved into a precursor (per-miRNA) of the stem-loop structure in the nucleus by Dicer Like protein (DCL1). Mature plant miRNA:miRNA* duplex excised form pre-miRNA by DCL1 and then exported to the cytoplasm [1, 3, 4]. The mature miRNA strand of duplex strand is loaded into an RNA-induced silencing complex (RISC) that normally contains an Argonaute (AGO) protein in the cytoplasm, then leads the RISC to cause site-specific repression or cleavage of the mRNA targets $[5,6]$. The other strand of the duplex, called miRNA*, is degraded [7]. Most plant miRNAs recognize their target mRNAs through homology-dependent mechanism and negatively regulate their expression by guiding cleavage or translation inhibition [4, 8, 9], furthermore some miRNAs may activate their targets, especially metazoan miRNAs [10-12].

Samiliar to miRNAs, siRNAs also have significant functions in plants. PhasiRNA is a class of secondary siRNAs that are generated precisely in 21 or 24 nt phased pattern initiated at a specific position due to miRNA guided activity. A type of phasiRNAs are trans-acting siRNAs (tasiRNAs) because they repress their target transcripts from other loci of the genome at post-transcriptional level [13]. The primary transcripts of tasiRNAs are non-coding and used to generate double strand RNAs (dsRNAs) by RDR6 (RNA-dependent RNA polymerase 6) [14]. The dsRNAs are then cleaved by DCL4 to form phased $21 \mathrm{nt}$ segments [15-17] or by DCL5 to form 24 nt phased segments as shown in rice $[18,19]$. The precise phasing of tasiRNAs is guided by miRNAs [15] through either two [20] or one [18, 21-24] miRNA binding site on the primary tasiRNA transcripts. Four families of tasiRNA loci, named TAS1 to TAS4, have been identified in Arabidopsis thaliana $[15,17,25]$. Recent studies suggest that some coding genes, especially PPR $[17,24,26]$, NB-LRR disease resistance proteins [24, 27-31], MYB transcription factors $[24,32,33]$, also generate phasiRNAs, and their corresponding loci are called as PHAS loci [27]. These PHAS loci are also triggered by one or two miRNA binding sites $[14,27]$. TAS3 derived tasiARFs are the only phasiRNAs that have been validated to target ARF genes in trans $[15,20,24]$. The functions of most phasiRNAs are still largely unknown [14].

Rose is a generic name for a variety of rosa. Rosa rugosa Thunb. (R. rugosa) (old rose), originated in China, belongs to the Rosa family. It is a cash crop important for its fragrant and beautiful flowers [34]. In the past century, $R$. rugosa has emerged as one of the most important crops in the floriculture industry worldwide and cut $R$. rugosa accounts for nearly one third of cut flower trade in Europe [35]. In addition, $R$. rugosa possesses some unique morphological and physiological features, that include highly divergent flower colors, shapes and volatiles and recurrent flowering, which are unable to be studied in other model plant systems, like tobacco and Arabidopsis thaliana [36]. $R$. rugosa flowers are mainly used in food and refined $R$. rugosa otto. $R$. rugosa otto is widely used in cosmetics, foodstuff, fine chemicals and other industries. Although $R$. rugosa is very important, there are only a few studies that reported sRNAs in $R$. rugosa [34, 37].

Flowers are the most important organs in $R$. rugosa for the economic and ornamental values. Therefore, we preferentially chose to study the miRNAs and phasiRNAs in $R$. rugosa flowers. We sequenced 10 small RNA libraries from 5 tissues, i.e., roots, petals, pollens, stamens and leaves and 4 RNA-seq libraries from leaves and petals of $R$. rugosa. We found that 2000 genes have significantly different expression levels in leaves and petals of $R$. rugosa. These genes are mainly involved in different metabolic processes and photosynthesis related pathways. Comprehensive analyses of these small RNA profiles lead to identification of 25 pre-miRNAs of which 24 have not been reported, 22 novel miRNAs, 339 PHAS loci encoding 21 nt phasiRNAs, and 49 PHAS loci encoding 24 nt phasiRNAs. A degradome profile of $R$. rugosa leaf was generated for identifying targets of miRNAs and phasiRNAs. Totally, more than 19,000 putative targets for conserved miRNAs and TAS3-derived tasiRNAs were identified in the analysis. At least 592 targets have shown significant accumulation of degradome reads corresponding to the identified miRNA complementary sites. These results clearly deepen our understanding about small RNA guided gene regulations in $R$. rugosa.

\section{Methods}

\section{Materials and small RNA sequencing profiles}

The $R$. rugosa plants were grown in Kunming University of Science and Technology, Yunnan, China. We collected ten samples from the roots, leaves, pollen, petals, and stamens of $R$. rugosa without treatment and frozen with liquid nitrogen immediately (Additional file 1: Table S1). Before RNAs were extracted, these 10 samples were stored at $80^{\circ} \mathrm{C}$. According to the manufacturer's protocol, we used the Trizol reagent to extracte total RNAs from samples. Based on the ratio of the optical density at $260 \mathrm{~nm}$ to that at $280 \mathrm{~nm}(\mathrm{OD} 260 / 280)$, the integrities of RNAs were checked by using an ultraviolet spectrophotometer (Hoefer, MA, USA). And then in view of visual comparison of the $18 \mathrm{~S}$ and $28 \mathrm{~S}$ ribosomal RNAs, the integrities 
of RNAs were also assessed by electrophoresis in a denaturing formaldehyde agarose gel. The total quantities of RNA samples with OD260/280 between 1.8 and 2.0 were examined. Samples with at least $20 \mu \mathrm{g}$ were selected for preparation of sRNA sequencing libraries. $20 \mu \mathrm{g}$ total RNAs dissolved in $35 \mu \mathrm{l}$ were delivered to the sequencing facility. For small RNA-seq library generation, $1 \mu \mathrm{g}$ of total RNA was used to prepare a small RNA library according to the TruSeq Small RNA Sample Prep Kit protocol (Illumina, San Diego, USA). Briefly, specific 5' and 3' Illumina RNA adapters were sequentially added to small RNA molecules. After reverse transcribed and PCR amplified, adapter-ligated molecules with 15 to 30 nucleotides long were purified by using gel electrophoresis. The small libraries were then multiplexed and sequenced by using the Illumina HiSeq 2000 sequencer. The qualities of the obtained sRNA profiles were evaluated with the FASTQC program (https://www.bioinformatics. babraham.ac.uk/projects/fastqc/). The obtained small RNA profiles had been deposited to NCBI GEO database under the accession number, GSE110490.

\section{RNA-seq profiles of leaves and petals in $R$. rugosa}

Two petal and leaf samples of $R$. rugosa plants grown in Kunming University of Science and Technology, Yunnan, China were collected and put into liquid nitrogen immediately. Total RNAs of these 4 samples were extracted using the same protocol as above. The integrities of the RNAs were also checked using same protocols as above. The total RNAs of the 4 samples were sequenced using Illumina HiSeq 4000 sequencer with a $2 \times 150$ pair-end RNA-seq strategy. The obtained RNA-seq profiles were deposited into the NCBI GEO database under the accession number GSE113148.

These 4 RNA-seq profiles were assembled using Trinity (v2.0.6) [38] with the options of "-seqType fq max_memory 400G -min_contig_length 150 -CPU 30 -min_kmer_cov 3 -min_glue 3 -bfly_opts '-V 5 edge-thr $=0.1$-stderr"'. This results in a transcriptome with more than 370,000 transcripts. These transcripts were then aligned to NCBI nt/nr database with the options of "-e 1e-5 -m 8" to obtain their putative annotations.

The expression levels of the assembled transcripts were estimated by the align_and_estimate_abundance.pl program in the Trinity package using the options of “-seqType fq -est_method RSEM -aln_method bowtie -trinity_mode -prep_reference".

The expression levels of transcripts in leaf and petal samples were compared with edgeR [39]. The expression levels of transcripts were filtered to keep 37,278 transcripts with average expression levels of at least 10 FPKM and standard deviations of expression levels of at least 10 FPKM. Then, the expression levels of these 37,278 were used to perform a bi-clustering analysis using the pheatmap library in R. Transcripts with FDR values smaller than 0.05 were called as deregulated. The deregulated transcripts were used to analyzed enriched GO terms and KEGG pathways using KOBAS (version 3) [40].

\section{Identification of conserved and novel miRNAs}

By using the computational methods reported previously [41], we analyzed the small RNA libraries. Firstly, we filtered out raw reads containing more than five low scored nucleotides $(<25)$. After removing 3' adaptor sequences of the remaining reads and redundant sequences, we obtained unique small RNAs. Then we removed unique sequences which could be mapped to known repeats and non-coding RNAs (snRNAs, tRNAs, rRNAs, snoRNAs) by aligning them to databases, Rfam (r11) [42], NONCODE (v3.0) [43], Silva [44], The TIGR Plant Repeat Databases [45] and Repbase (r20) [46] using SOAP2 [47]. To calculate the frequencies of conserved miRNAs, we mapped remaining reads to the miRBase database (r21) [48]. We also counted and summarized the numbers of reads that were mapped to different types of molecules (Additional file 1: Table S2).

The remaining unique sequences that could be mapped to the transcriptome, but not to pre-miRNAs, ncRNAs and repeats were aligned to the self-assembled $R$. rugosa transcriptome by SOAP2. The flanking sequences (downand up-stream) of the matched loci were used to predict secondary structures using RNAfold [49]. We selected the structures that satisfy the following criteria as putative precursors: at least 18 paired nucleotides, only one central loops in the hairpin structure and a folding energy of smaller than $-30 \mathrm{Kcal} / \mathrm{mol}$. The small RNA sequencing reads in the 10 sRNA-seq libraries were mapped to the obtained putative precursors. Finally, novel miRNA identification and annotation were strictly based on appearance of miRNA* and predictable fold back structures for the miRNA precursor sequences, as suggested by Meyers et al. [50].

In order to identify conversed miRNAs homologs in $R$. rugosa, we downloaded all mature miRNA sequences of plant species from the miRBase (v21) [48], and removed redundant sequences as described previously [13, 41]. Then these unique miRNA sequences were mapped to the $R$. rugosa cDNAs using BLASTN. Hits with no more than two mismatches were identified. The flanking regions (80nt, 130nt, 180nt down stream and upstream) of the mapped mature miRNAs were isolated and used to predict secondary structures using the RNAfold. The predicted fold-back structures were examined for the presence of miRNA on the same arm of the hairpin as the known family members from other plants. We use MIRcheck to further evaluate these precursor sequences. 
The precursors were selected which have $\leq 2$ bulged, $\leq 5$ mismatches, or asymmetrically unpaired nucleotides, and $\leq 3$ continuous mismatches within the mature miRNA.

Then the identified conserved miRNAs was compared with those reported previously [34,37]. If the sequence of a mature miRNA was identical to the previously reported sequence, the conserved miRNA was named the same as in the previous report. The remaining conserved miRNAs were named by using upper case MIR followed by the family name, and alphabetical letters in lower case if these have not been reported earlier.

\section{Analysis of miRNA expression patterns in different tissues}

To analyze the expression patterns of miRNAs in different tissues, we used edgeR [39] to compare the RPTM (Reads Per Ten Million sequencing tags) of mature miRNAs from different tissues. miRNAs with FDR (False Discovery Rate) less than 0.05 were considered as significantly deregulated miRNAs in different tissues.

miRNA with abundances of at least 5 RPTM in at least two of the 10 samples and standard deviation of at least 1 were selected to perform hierarchical clustering. The normalized RPTM values plus one were log scaled and applied to the pheatmap function in the pheatmap library in $\mathrm{R}$ to perform bi-clustering. miRNAs with RPTM mean of at least 5 in 10 samples were used to perform Principle Component Analysis (PCA). The RPTM values plus one for these miRNAs were log-scaled and used to perform PCA by the procmp function in $R$.

\section{Identification of TAS3 loci in $R$. rugosa}

As reported previously $[51,52]$, tasiRNAs that were produced from TAS3 genes in Arabidopsis and rice were collected. These tasiRNAs were aligned to the self-assembled $R$. rugosa cDNA database with up to two mismatches allowed. Both the upstream and downstream $250 \mathrm{bp}$ sequences of matched loci were cut out form cDNA.

\section{Identification of PHAS loci in R. rugosa}

We used 10 small RNA profiles generated in this study to predict PHAS loci and phasiRNAs. The method for predicting PHAS loci and phasiRNAs were described previously $[13,41,53]$. The unique sequences in the small RNA libraries were aligned to The TIGR Plant Repeat Databases [45] and Repbase (r20) [46] to remove sRNAs mapped to repeats, then the remaining sRNAs were mapped to the cDNA of $R$. rugosa using SOAP2 [47]. In order to scan the cDNA sequences using a window of $210 \mathrm{nt}$ or $240 \mathrm{nt}$ (ten $21 \mathrm{nt}$ or $24 \mathrm{nt}$, respectively) respectively, a self-written program was used. Because the existence of two-nucleotide overhang at the 3'-end of siRNA duplex, a two-nucleotide positive offset was used to calculate the positions of siRNAs on the anti-sense strand [17, 24, 26, 27]. Then a $P$-value was calculated for each of the windows using a Hypergeometric test [26]. And a phase score was calculated for each position of $\mathrm{cDNA}$ sequences using the method in [54].

The window with a $P$-value less than 0.05 was extended $100 \mathrm{bp}$ at both 5 - and $3^{\prime}$-ends, then the overlapped windows were merged. The $P$-values of the combined windows were used to calculate the false positive rates using the method in [55]. The merged windows with a maximal phase scores of more than 5 and FDR values less than 0.05 were reported as PHAS loci. The predicted PHAS loci were named with the contig names of self-assembled cDNAs and a unique serial number for each contig. "P21" and "P24" were added at the beginning of the predicted PHAS loci encoding 21 and 24 nt phasiRNAs. The neighboring PHAS loci were predicted as PHAS clusters if the distances between individual PHAS loci were smaller than 2000 base pairs. The phased siRNAs of the predicted PHAS loci were reported as phasiRNAs. The phasiRNAs of a PHAS loci were named by adding siR and a unique serial number to the name of the PHAS loci $[13,41,53]$.

\section{Degradome sequencing}

A leaf sample was frozen in liquid nitrogen immediately after harvesting form a $R$. rugosa plant grown in Kunming University of Science and Technology, Kunming, Yunnan, China. According to the manufacturer's protocol, we used the Trizol reagent to extracte total RNAs from samples. The integrity of the RNA was checked with an ultraviolet spectrophotometry and Agilent 2100 BioAnalyzer. For constructing degradome library, $20 \mu \mathrm{g}$ of total RNA was annealed with biotinylated random primers. These biotintagged RNA fragments were then captured on streptavidin. Then the RNAs which contain 5' -monophosphates were ligated with a 5 ' adaptor. After reverse transcription and PCR amplification, libraries were sequenced using the 5 ' adapter on an Illumina HiSeq 2000 sequencer with a single-end mode of 47 base pairs. The sequenced reads represented the 5' ends of the sliced RNA fragments that were ligated with the adapters. The obtained degradome profile had been deposited to the NCBI GEO database under the accession number, GSE110494.

The quality of the obtained degradome profile was evaluated with the FASTQC program. The obtained degradome profile was processed with a self-developed pipeline. Firstly, low quality reads that have low scored nucleotides $(<30)$ in the first 25 nucleotides were removed from the raw degradome sequencing profile. Then the first twenty nucleotides were cut out from the remaining reads for later analysis. Next, we removed redundant sequences and calculated the frequencies of the unique sequences. Similar to sRNA profiles, unique sequences were aligned to cDNA of $R$. rugosa, Rfam (r11) [42], NONCODE (v3.0) [43], Silva [44], The TIGR Plant Repeat Databases [45] and Repbase (r20) [46] using 
SOAP2 [47] to calculate the numbers of reads and unique sequences mapped to different categories of molecules as well as repeats. The SOAP2 alignment result of mapping degradome sequences to $R$. rugosa cDNA was used to identify targets of miRNAs and siRNAs.

\section{Identification of miRNA/siRNA targets in R. rugosa}

To predict the targets of miRNAs and tasiRNAs, we used the SeqTar pipeline [56]. For conserved miRNAs and tasiRNAs, targets with less than four mismatched nucleotides to miRNAs/tasiRNAs were used for later analysis. For novel miRNAs, targets with at least one valid read and no more than four mismatches were selected for subsequent analysis.

Similarly, the SeqTar algorithm was used to predict targets of phasiRNAs. Only targets that satisfy the following two conditions were used for later analysis: targets that have no mismatches or at least 1 valid degradome read and no more than 3 mismatches.

\section{Identifying miRNA complementary sites on PHAS loci}

The miRNA complementary sites on the original transcripts of PHAS loci were predicted with SeqTar. For conserved miRNAs, the targets that have at least one valid read, i.e., read started at the 9th to 11th positions of a miRNA binding site (as defined in [56]), or targets that have less than 4 mismatches were used for further analysis.

\section{Results and discussion}

\section{RNA-seq profiles of leaves and petals of $R$. rugosa}

Two petal and leaf samples of $R$. rugosa plants grown in Kunming University of Science and Technology, Yunnan, China were collected and put into liquid nitrogen immediately. Four RNA-seq profiles were generated using Illumina HiSeq 4000 sequencer. The qualities of these RNAseq profiles are good (Additional file 2: Figure S1) after examining the scores of sequences with FASTQC. The RNA-seq profiles were assembled using Trinity (v2.0.6) [38]. We generated a self-assembled $R$. rugosa transcriptome with more than 37,000 segments. Then we use Trinity's own program TrinityStats.pl to count the number and size of components and transcripts, N50, etc. As the result, we got 302,082 Trinity genes and 370,847 Trinity transcripts. The Contig N50 is 869 . In order to derive annotation, we aligned this self-assembled transcriptome to NCBI nt/nr database (see "Methods" for details).

\section{Deregulated genes in leaves and petals of $R$. rugosa}

We calculated the normalized expression levels (Fragments Per Kilo basepairs per Million fragments mapped) of genes for four samples (Additional file 1: Table S3). Then, we performed Hierarchical Clustering based on the normalized frequencies of genes (Fig. 1a). We compared the abundances of leaves and petals with edgeR (Additional file 1: Table S4). We have identified 1811 deregulated genes. Among them, 1015 and 796 genes are upregulated and downregulated in petals, respectively (see Fig. 1b). These deregulated genes were used to analyze the enriched GO terms and KEGG pathways with KOBAS (version 3) [40]. The results show that the upregulated genes in petals are mainly involved in various metabolic processes and oxidoreductase (Fig. 2a), while the downregulated genes in petals, i.e., upregulated genes in leaves, are mainly involved in Response to Stresses, Oxidation-reduction Process, Response to Light Stimulus, and Binding (Fig. 2b). As shown in Fig. 2c and d, many upregualted and downregulated genes in petals are involved in Metabolic Pathway. Several pathways related photosynthesis, such as Photosynthesis - antenna proteins and Carbon fixation in photosynthetic organisms, are enriched in genes upregulated in leaves, i.e, downregulated in petals (Fig. 2d). These results are consistent with the types of the tissues, since the photosynthesis pathway is mainly performed in leaves.

Small RNA sequencing profiles of different $R$. rugosa tissues We collected the samples of roots, petals, pollens, stamens, leaves from $R$. rugosa that were grown in Kunming, Yunnan, China, without special treatments. Ten small RNA-seq (sRNA-seq) profiles, with two replicates for each of the five tissues, i.e., roots, petals, pollens, stamens, and leaves of $R$. rugosa, were generated using Illumina HiSeq 2000 sequencer (Additional file 1: Table S1). The qualities of these sRNA-seq profiles are good (Additional file 2: Figure S2) after examining the scores of sequences with FASTQC. Totally, we obtained 78,515,641 total reads represented by 25,456,782 unique small RNAs (Additional file 1: Table S2). The sRNA-seq profiles were aligned to the self-assembled transcriptome of $R$. rugosa, precursors of miRNAs in the miRBase (v21), other non-coding RNAs besides miRNAs, repeat elements and genome of $R$. rugosa with SOAP2 (see details in Methods) to obtain the distributions of sRNA reads (Additional file 2: Figure S3 and Additional file 1: Table S2).

\section{Identification of conserved miRNAs}

A total of 321 mature conserved miRNAs from other species were detected in our sRNA-seq profiles (Additional file 1: Table S5). A previous study [37] reported 242 conserved mature miRNAs, identified from flowers of three modern rose cultivars and Rosa rugosa. Seventy three of the 242 miRNAs identified in this previous study were found in our libraries (Fig. 3a). In addition, we identified 248 conserved miRNAs that were not reported in their study (Fig. 3a). By aligning these mature miRNAs to the self-assembled $R$. rugosa transcriptome, we identified 25 precursors of conserved 

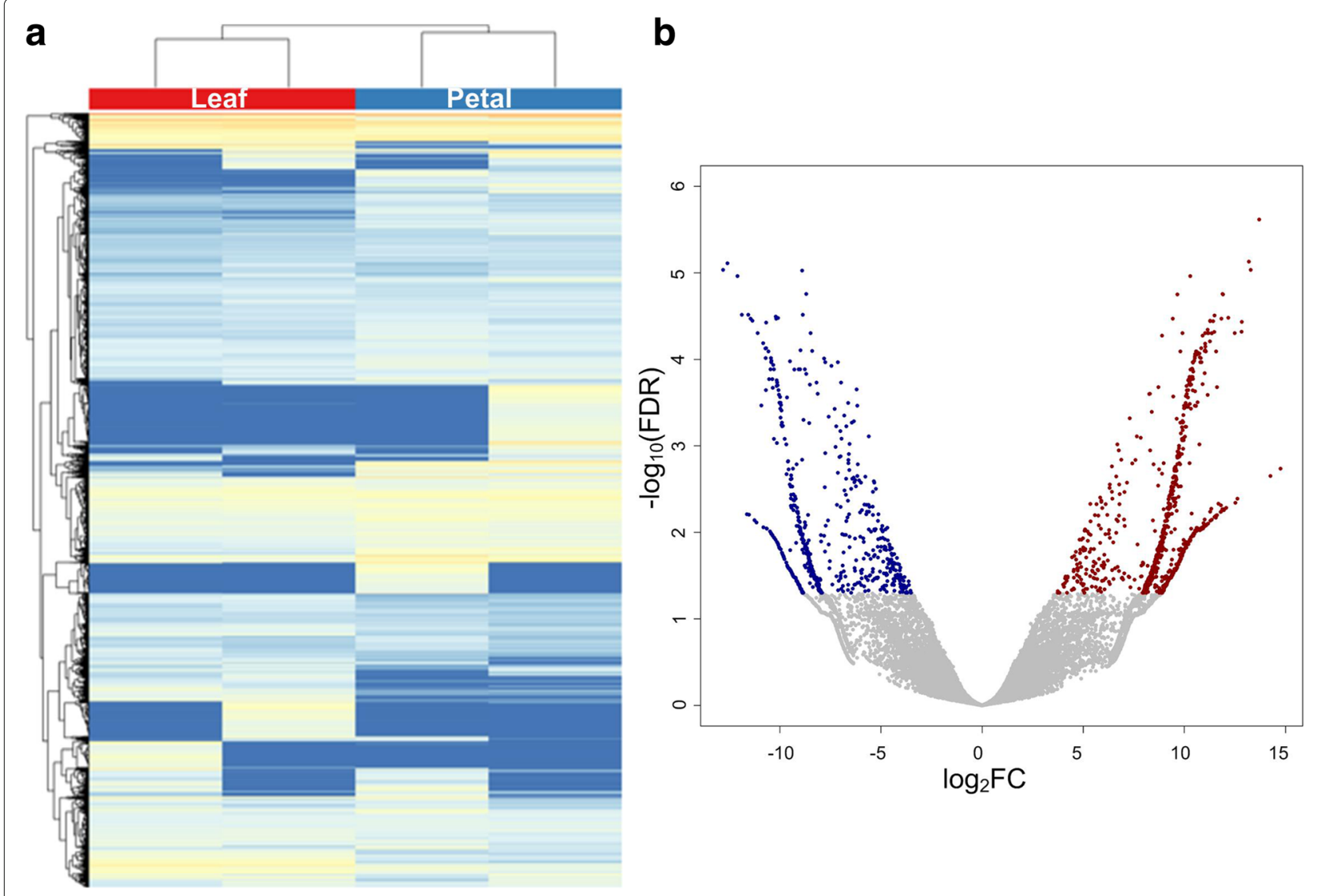

Fig. 1 Expression patterns of genes in R. rugosa leaves and petals. a The bi-clustering of genes expression profiles in different tissues of $R$. rugosa RNA-Seq profiles. The values shown are the $\log _{2}($ FPTM+1) of the transcripts. b Deregulated genes when comparing their expression levels in petals to those in leaves. Up and downregulated genes in petals are shown in red and blue dots, respectively

miRNAs (Additional file 1: Table S6 and Fig. 3b). Among them, only the precursor of miR482 had been reported previously [34]. When compared to two model species, Arabidopsis thaliana and rice, we found 18 members that belong to 11 miRNA families that are highly conserved (Additional file 1: Table S7).

\section{Expression patterns of conserved miRNAs in different tissues}

Since we have constructed small RNA libraries from five different tissues of $R$. rugosa, we were able to analyze the different expression patterns of miRNAs in these tissues. Based on the frequencies of mature miRNAs after standardization, we performed PCA and Hierarchical Clustering. According to PCA and clustering results, it can be clearly seen that samples from the same tissue were clustered together, and samples from different tissues were distinguished. (Figure $3 \mathrm{c}$ and $3 \mathrm{~d}$ ). We also found that the expression levels of miRNAs are similar in the same tissues (Fig. 3d). These results indicate that miRNAs not only have important functions, but may also be involved in different physiological processes in different tissues.

To identify deregulated miRNAs in different tissues, we compared the expression levels of conserved miRNAs in samples from root, leaf, petal, stamen and pollen with edgeR [39] (Additional file 1: Tables S8 to S17). According to the results of differential expression analysis, we found 12 to 130 deregulated miRNAs between different tissues (Additional file 1: Table S18). For examples, MIR167j has higher expression levels in pollens, leaves, stamens than in the roots and petals; MIR319g has higher expression level in roots, petals, pollens and stamens than in leaves; and MIR156c has higher expression levels in pollens than roots, petals, and stamens.

\section{Discovery of novel miRNAs in $R$. rugosa}

We identified 22 putative novel miRNAs in $R$. rugosa (Additional file 1: Table S19). Four of the 22 putative novel miRNAs are shown in Additional file 2: Figure S4. Both mature miRNA and miRNA* were detected in our sequencing libraries (Additional file 2: Figure S4a). The 

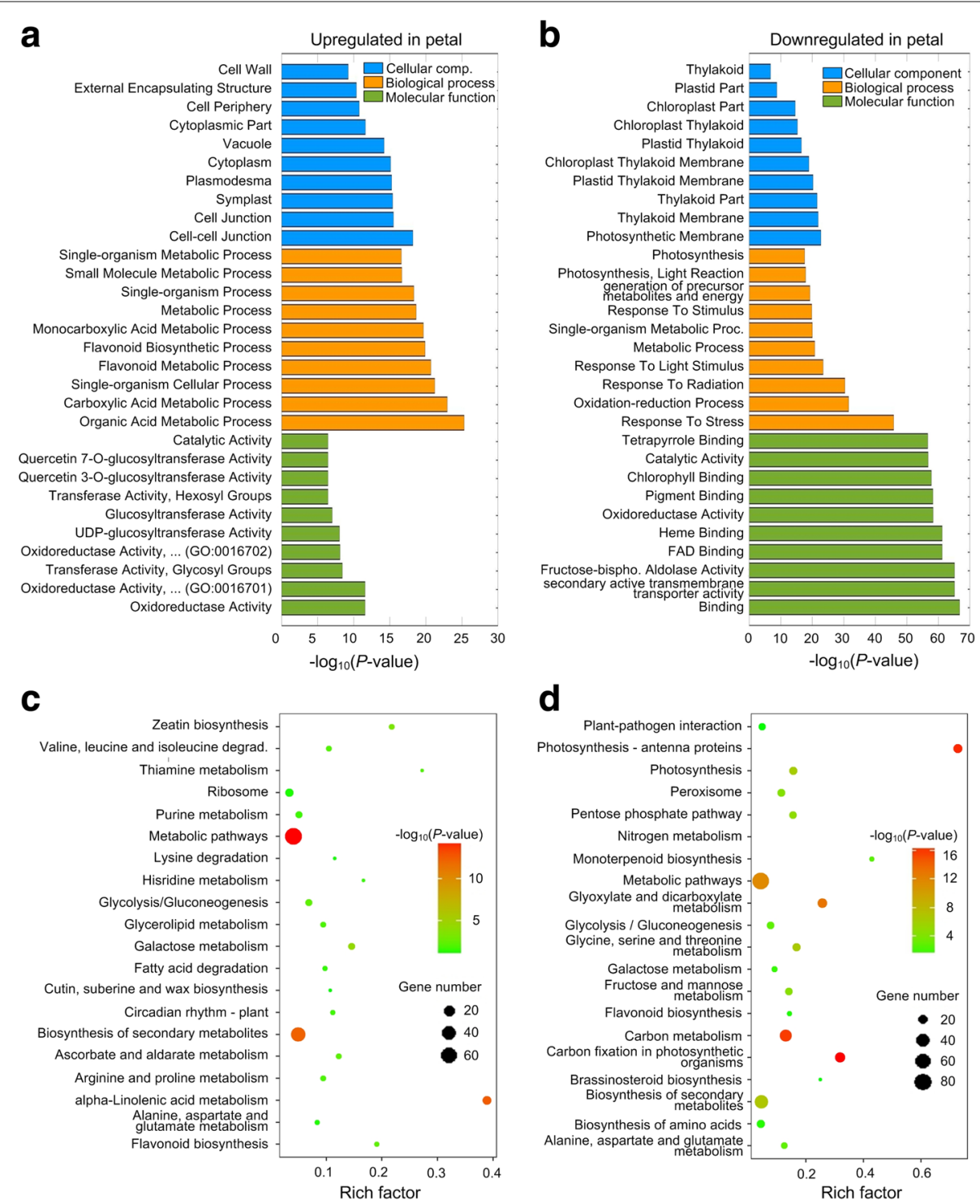

Fig. 2 Enriched GO terms and KEGG pathways in deregulated genes of leaf and petal samples of R. rugosa. $\mathbf{a}$ GO terms of upregulated genes in petals. b GO terms of downregulated genes in petals. $\mathbf{c}$ Enriched KEGG pathways of upregulated genes in petals. d Enriched KEGG pathways of downregulated genes in petals. In Part (c) and (d), the Rich Factor is calculated by dividing the number of input genes with the KEGG pathway to the total number of genes with the same pathway

mature miRNAs demonstrated clear accumulated abundances than other regions of the pre-miRNAs in different sRNA sequencing libraries (Additional file 2: Figure S4b). These evidences suggest that these miRNAs are real miRNAs according to the criteria for annotating miRNAs [50].

\section{Identifying miRNA targets in $R$. rugosa}

We producted a degradome profile with over 23 million reads (Additional file 1: Table S20) to identify miRNA targets. By running FASTQC, we found that most nucleotides of the degradome reads have sequencing scores of 35 or higher (Additional file 2: Figure S5). We used the SeqTar algorithm [56] to identify targets for conserved miRNAs from the obtained degradome profile of $R$. rugosa leaf. This analysis revealed more than 19,000 putative targets for conserved miRNA families (Additional file 1: Table S21). 592 of these targets have significant accumulation of reads at the identified miRNA complementary sites with at least 3 valid reads and $P$-value of valid reads smaller than 0.05 (Additional file 1: Table S21). 


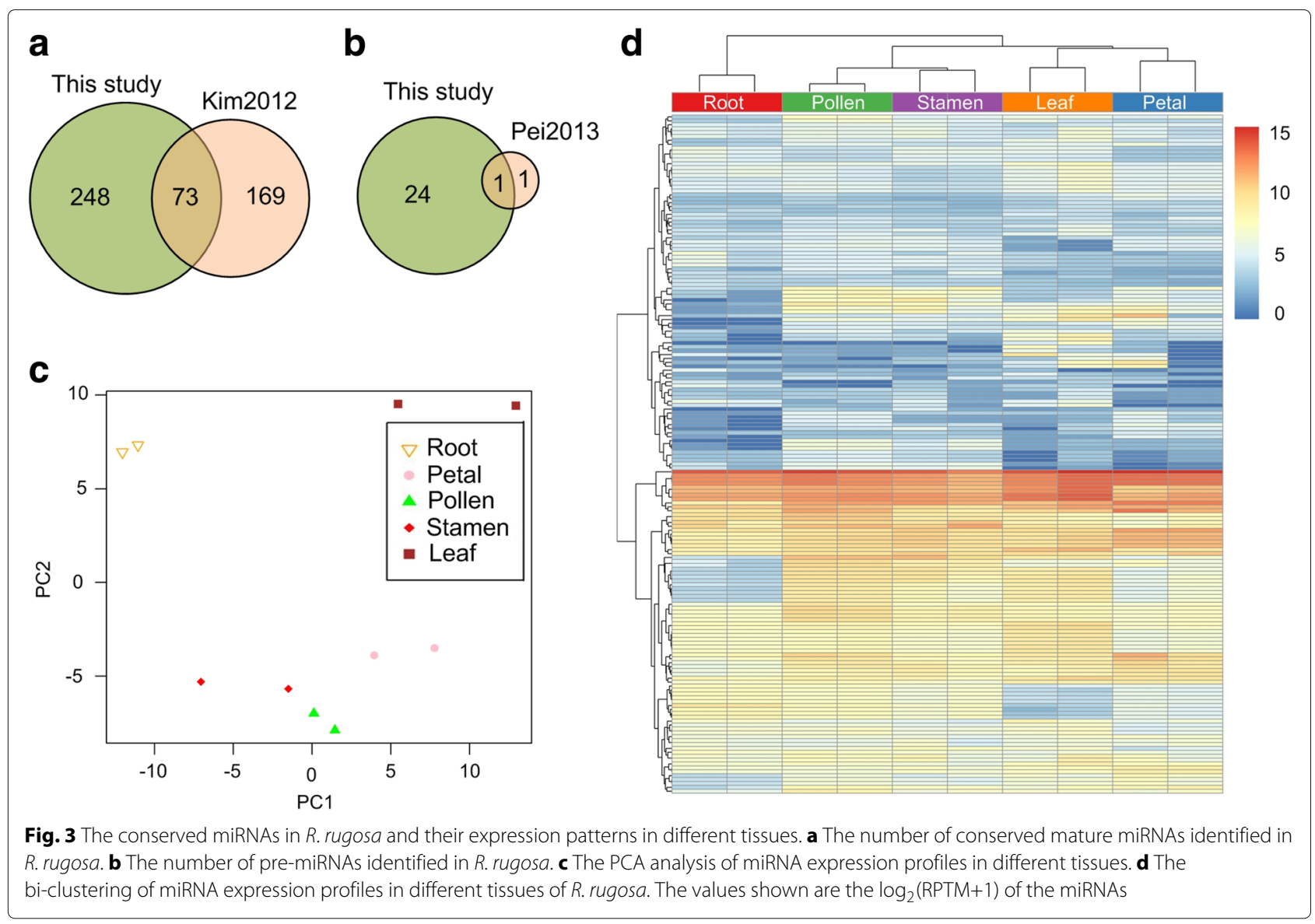

We compared the number of conserved target genes for 26 highly conserved miRNA families to those in Arabidopsis and rice. Totally, we found 81 conserved targets for these 26 miRNA families (Additional file 1: Table S22). Nine of these targets were shown in Fig. 4 and 12 more were shown in Additional file 2: Figure S6. Among these targets identified in degradome analysis, only one AP2 gene targeted by miR172 (TR68272|c1_g1_i3) was validated in a previous study [37].

\section{PHAS loci in R. rugosa}

The sRNA profiles of 10 samples in this study were aligned to self-assembled $R$. rugosa transcriptome using SOAP2 [47]. Then, we used an in-house written program [57] to predict PHAS loci and phasiRNAs from the alignment results of SOAP2 based on methods described earlier (see Methods for details). We predicted three hundred and thirty nine 21 nt PHAS loci by using a phase score threshold of 5 and a multiple-test corrected $P<0.05$ (Additional file 1: Table S23). We aligned the obtained PHAS sequences to NCBI Nucleotide Collection (nr/nt) database and the TIGR Plant Repeat database to obtain putative annotation of the predicted PHAS loci (details are given in Additional file 1: Table S19). These PHAS loci were mainly derived from protein coding genes (Fig. 5a).

Similarly, by using a phase score threshold of 5 and a multiple-test corrected $P<0.05$, we predicted forty nine 24 nt PHAS (Additional file 1: Table S24). Most of 24 nt PHAS loci belong to unknown category in $R$. rugosa (Fig. 5b), which is different from the 21 nt PHAS loci. The second largest category of 24 nt PHAS loci is protein coding genes (Fig. 5b).

Taken together, the 339 and 49 PHAS loci encoding 2859 and 369 twenty one and twenty four nt phasiRNAs, respectively (Additional file 1: Tables S25 and S26, respectively). The putative targets of 21 and $24 \mathrm{nt}$ phasiRNAs are provided in Additional file 1: Tables S27 and S28, respectively. One of the 21 nt PHAS loci is a TAS3 gene shown in Fig. 6a. It is targeted by miR390 at two loci (Fig. 6b and c) around a conserved tasiRNA, named as TAS3a_D8(+), (the yellow part in Fig. 6a), using the naming method described earlier [15]. From degradome analysis, it was found that a few reads corresponding to these two miR390 complementary sites were generated. This locus generates numerous siRNAs (Fig. 6d). The conserved tasiRNA targets at least four ARF genes (Fig. 6f). Two of these 
a

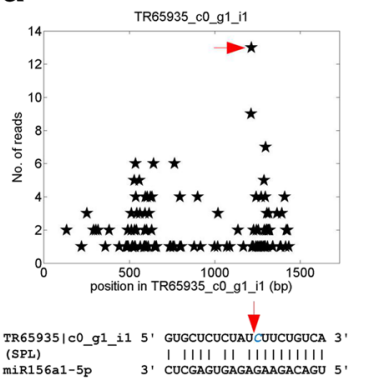

d

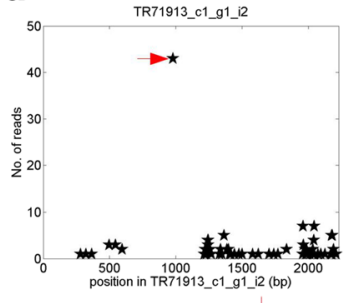

TR71913|c1_g1_i2 5' cugGAaUGAaGCCUGGUCCGG 3'

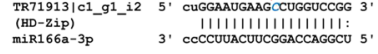

g

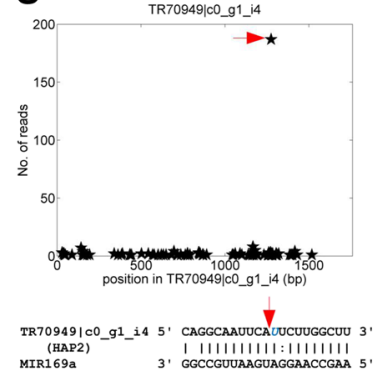

b

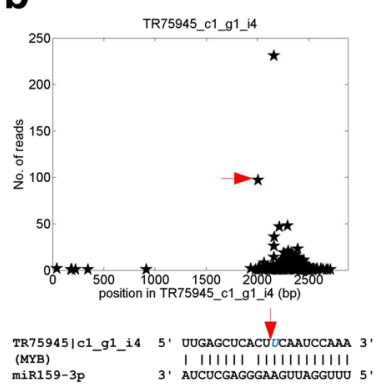

e

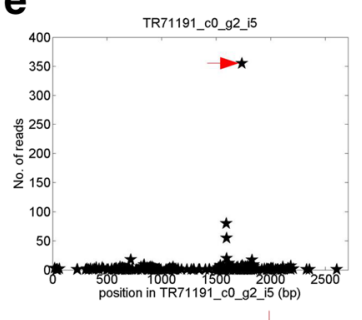

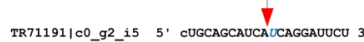

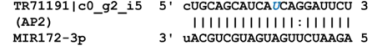

h

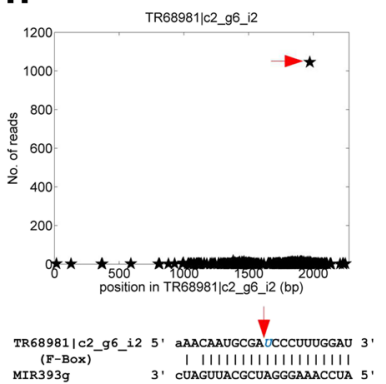

C

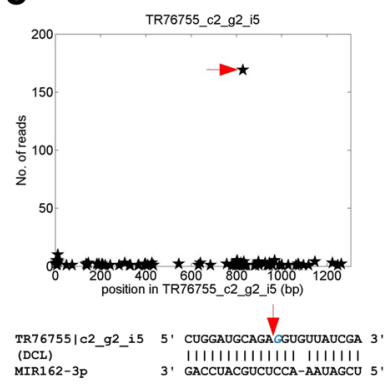

f

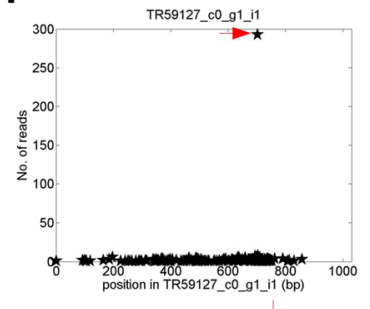

TR59127|c0_91_i1 5' AAGAGGUGA-CUGAGAACACA

$\begin{array}{ll}\text { TR59127|c0_g1_i1 } & \text { 5' AAGAGGUGA-CUGAGAACACA } \\ \text { (CSD) } & \text { AII IIIII IIIIIIIIII } \\ \text { MIR398a-3p } & \text { 3. UUCCCCACUGGACUCUGGUG } 5\end{array}$

i

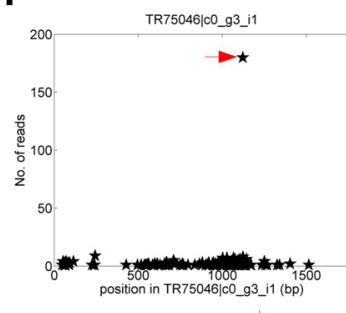

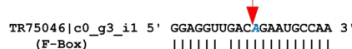

(F-Box)
MIR394b

Fig. 4 Some of the identified conserved targets of miRNAs in R. rugosa. The x-axis is the position on the transcript, and y-axis is the number of reads detected from a position. The arrows in the upper parts correspond to the positions pointed by the arrows of the same colors in the lower parts. $\mathbf{a}$ miR156a1-5p:TR65935|c0_g1_i1, a SPL gene. b miR159-3p:TR75945|c1_g1_i4, a MYB gene. c MIR162-3p:TR76755|c2_g2_i5, a DCL gene. d miR166a-3p:TR71913|c1_g1_i2, a HD-Zip gene. e MIR172-3p:TR71191|c0_g2_i5, an AP2 gene. f MIR398a-3p:TR59127|c0_g1_i1, a CSD gene. g MIR169a:TR70949|c0_g1_i4, a HAP2 gene. h MIR393g:TR68981|c2_g6_i2, a F-Box gene. i MIR394b:TR75046|c0_g3_i1, a F-Box gene

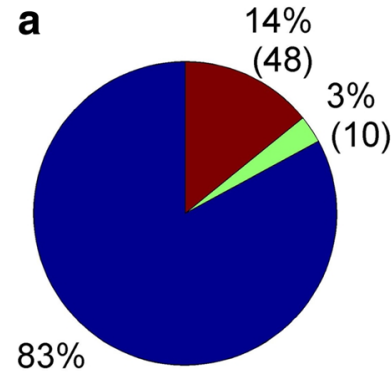

(281)

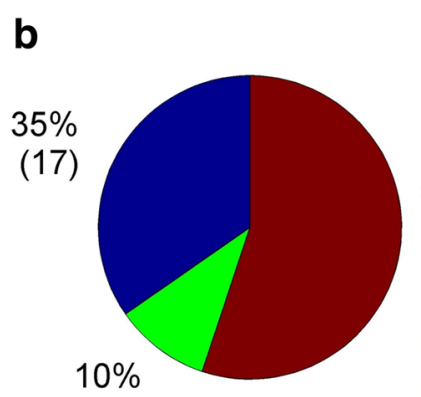

(5)
$55 \%$

(27)

protein coding genes

repeats

unknown

Fig. 5 The types of molecules of the identified PHAS loci in R. rugosa. a The categories of $21 \mathrm{nt}$ PHAS loci. $\mathbf{b}$ The categories of $24 \mathrm{nt}$ PHAS loci 


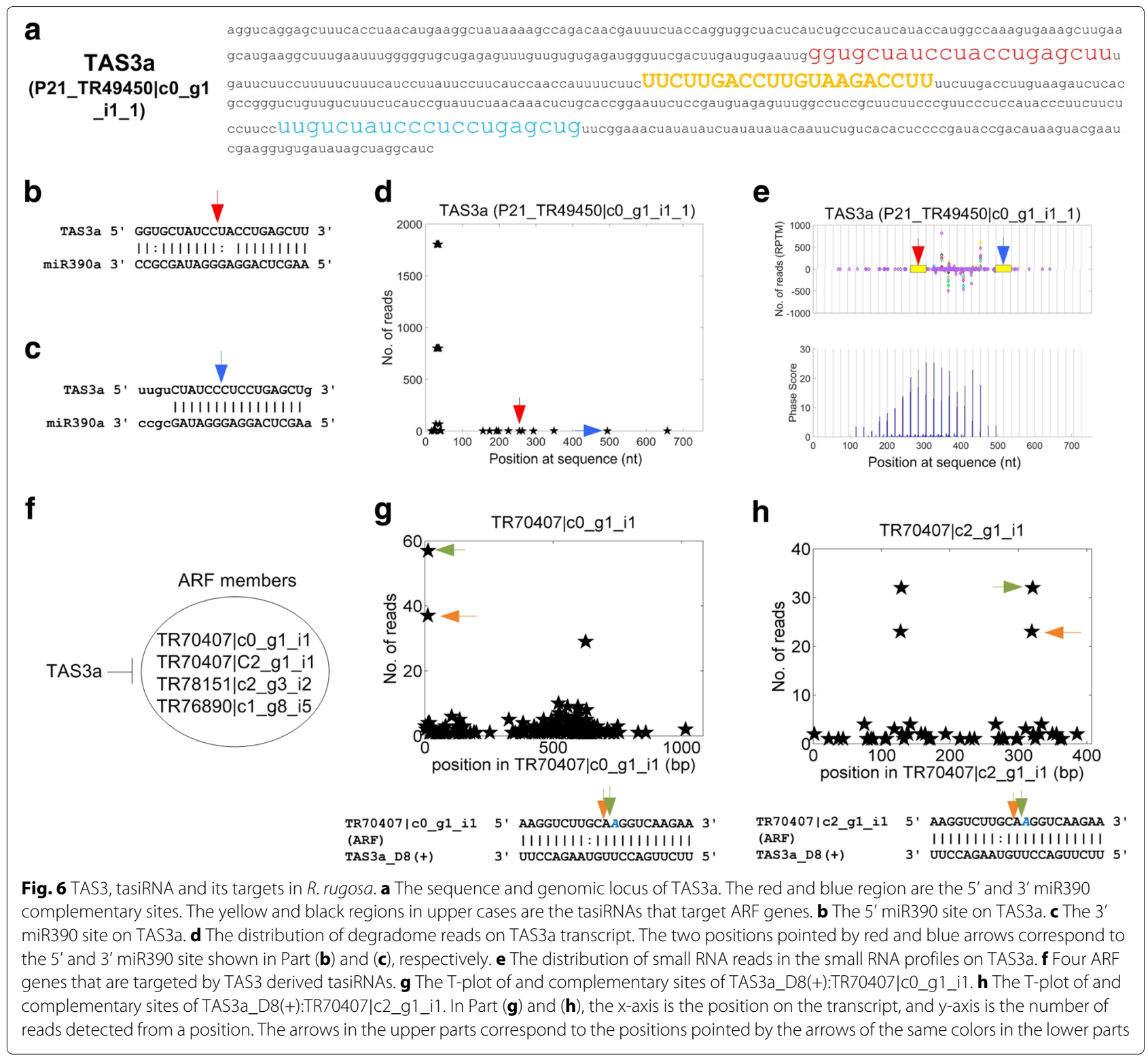

ARF targets are also validated by the degradome analysis (Fig. $6 \mathrm{~g}$ and $\mathrm{h}$ ).

Since miRNAs play key roles in the generation of phasiRNAs, we identified miRNA triggers for these PHAS loci on both strands by using the degradome sequencing profile and the SeqTar algorithm. The putative miRNA triggers of PHAS loci are also listed in Additional file 1: Table S23.

It is worth mentioning that miR482 potentially triggers the generations of phasiRNAs from more than 20 NBLRR genes (Additional file 1: Table S23). Two of them are shown in Fig. 7. From Fig. 7a, c and e, it is shown that miR482-3p triggers the generations of siRNAs from the centers of the complementary sites. Meanwhile, as indicated in Fig. 7b, d and f, miR482-3p could induce strong cleavages on these PHAS transcripts.

\section{Conclusion}

$R$. rugosa is an important crop plant for economical and floricultural reasons. However, there are only few studies that reported sRNA profiles in this important plant species. We systematically sequenced and analyzed 10 small RNA libraries from five different tissues of $R$. rugosa from which 321 conserved mature miRNAs were detected. We also identified 25 pre-miRNAs, 339 PHAS loci encoding $21 \mathrm{nt}$ phasiRNAs, and 49 PHAS loci encoding $24 \mathrm{nt}$ phasiRNAs. We also identified 22 novel miRNAs. By analyzing a degradome library, more than 500 reliable targets of conserved miRNAs or tasiRNAs were identified. Furthermore, our results suggest that approximately 20 putative NB-LRR genes are targeted by miR482-3p to generate phasiRNAs in $R$. rugosa. The RNA-seq profiles of leaves and petals suggest that thousands of genes have 
a
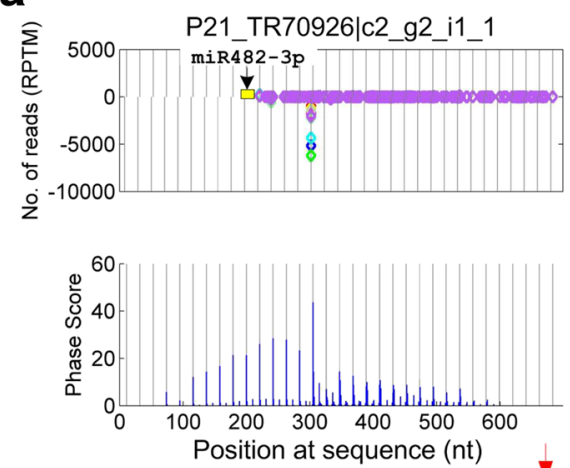

P21 TR70926/C2 g2 i1 1 , GGAAUGGAGGCGUUGGUAGA ${ }^{\text {P21_TR70926/c2 }}$

miR482-3p

3. CCUUACCCUCCGUAUCCGUUCU 5

C
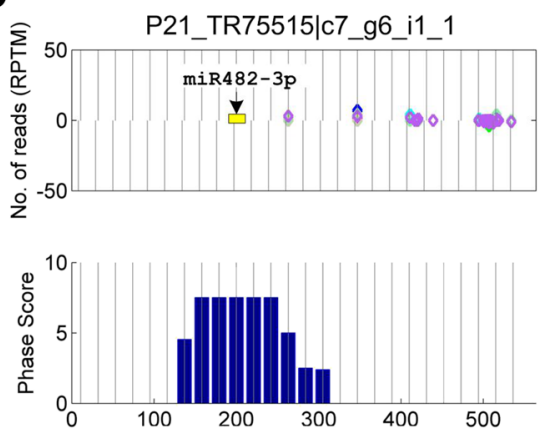

Position at sequence (nt)

d

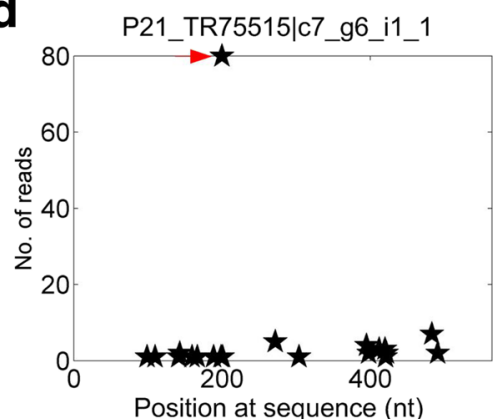

Position at sequence (nt)

b

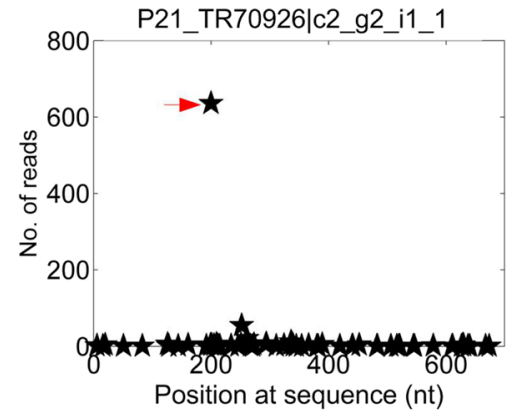

Position at sequence (nt)

Position at sequence (nt) $42 \mathrm{nt}$

|1||||||||||1:1 |||||||

e
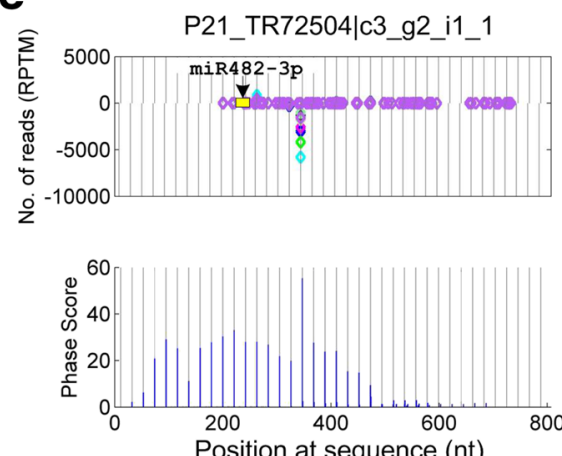

f

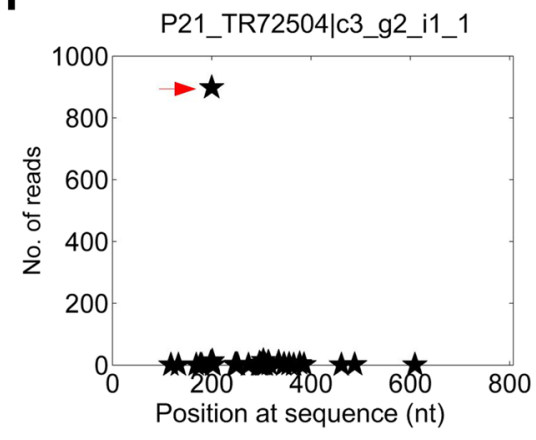

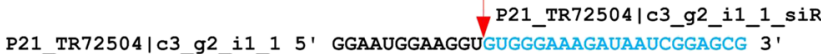

||||||| |||::1:1| ||||

miR482-3p 3' CCUUACCCUCCGUAUCCGUUCU 5
}

Fig. 7 Three PHAS loci from NB-LRR genes that are triggered by miR482. a The distribution of $21 \mathrm{nt}$ siRNAs and Phase Scores on P21_TR70926|c2_g2_i1_1. b The T-plot of and complementary sites of miR482a-3p:P21_TR70926|c2_g2_i1_1. c The distribution of $21 \mathrm{nt}$ siRNAs and Phase Scores on P21_TR75515|c7_g6_i1_1.d T The T-plot of and complementary sites of miR482a-3p:P21_TR75515|c7_g6_i1_1. e The distribution of 21 nt siRNAs and Phase Scores on P21_TR72504|c3_g2_i1_1.ffThe T-plot of and complementary sites of miR482a-3p:P21_TR72504|c3_g2_i1_1. In Part (a), (c) and (e), the vertical gray lines with distances of $21 \mathrm{nt}$ are the phased positions from the position with the highest phase scores of the PHAS loci. The yellow boxes in the read distribution panel represent the miR482a-3p complementary sites. Sites pointed by miRNAs from above and under zero read line means miRNAs complement to the plus and minus strand of the predicted PHAS loci, respectively. The predicted miR482a-3p complementary sites are shown below the phase score panel. The blue region after the complementary sites are one of the phasiRNAs generated from the PHAS loci 
shown significantly different expression levels in these two tissues of $R$. rugosa, and these genes are enriched in GO terms and KEGG pathways related to metabolic processes and photosynthesis. These results significantly improved our knowledge of sRNA guided gene regulations in R. rugosa.

\section{Additional files}

Additional file 1: This is an MS Excel file. This file includes 28 supplementary tables. (XLSX $43750 \mathrm{~kb}$ )

Additional file 2: This is a pdf file. This file includes 6 supplementary figures. (DOCX $1106 \mathrm{~kb}$ )

\begin{abstract}
Abbreviations
AGO: Argonaute; AP2: Adipocyte protein 2; ARF: Auxin response factor; CC: Correlation coefficient; cDNA: Complementary DNA; CSD: Copper-zinc superoxide dismutase; DCL: Dicer like; DCL1: Dicer like-1; DCL4: Dicer like-4; DCL5: Dicer like-5; dsRNAs: double strand RNAs; FDR: False discovery rate; FPKM: Fragments per kilobase million sequencing tags; GO: Gene ontology; HAP2: HAPLESS2; HD-Zip: Homeodomain leucine zipper family of transcription factors; KEGG: Kyoto encyclopedia of genes and genomes; logFC: log2FoldChange; miRNAs: microRNAs; mRNAs: message RNAs; MYB: Myeloblastosis family of transcription factors; NB-LRR: Nucleoside-binding site leucine rich repeat genes; ncRNAs: non-coding RNAs; PCA: Principle component analysis; phasiRNAs: phased small interfering RNAs; PPR: Pentatricopeptide repeats; pre-miRNAs: precursor microRNAs; RDR6: RNA-dependent RNApolymerase6; RISC: RNA-induced silencing complex; RNA-seq: RNA sequencing; RPTM: Reads per ten million sequencing tags; SeqTar: SEQuencing-based sRNA TARget prediction siRNAs: Small interfering RNAs; siRNAs: small interfering RNAs; SOAP: Short oligonuclotide analysis program; SPL: SPOROCYTELESS; sRNA: small RNA; sRNA-seq: small RNA-seq; tasiRNAs: trans-acting small interfering RNAs
\end{abstract}

\section{Acknowledgements}

Not applicable.

\section{Funding}

Funding for the publication of this article was provided by a grant (No. 31460295) of National Natural Science Foundation of China (http://www.nsfc. gov.cn/). The research was supported in part by two grants (No. 31460295 and 31760314) of National Natural Science Foundation of China (http://www.nsfc. gov.cn/) and a grant (No. SKLGE-1511) of the Open Research Funds of the State Key Laboratory of Genetic Engineering, Fudan University, China, to YZ.

\section{Availability of data and materials}

The obtained small RNA sequencing profiles were deposited to the NCBI GEO database under the accession number GSE110490 (https://www.ncbi.nlm.nih. gov/geo/query/acc.cgi?acc=GSE110490). The obtained degradome sequencing profile was deposited to the NCBI GEO database under the accession number GSE1 10494 (https://www.ncbi.nlm.nih.gov/geo/query/acc. cgi?acc=GSE1 10494). The obtained RNA-seq profiles were deposited into the NCBI GEO database under the accession number GSE113148 (https://www. ncbi.nlm.nih.gov/geo/query/acc.cgi?acc=GSE113148).

\section{About this supplement}

This article has been published as part of BMC Genomics, Volume 19 Supplement 9, 2018: 17th International Conference on Bioinformatics (InCOB 2018): genomics. The full contents of the supplement are available at https://bmcgenomics. biomedcentral.com/articles/supplements/volume-19-supplement-9.

\section{Authors' contributions}

YZ conceived and designed the research. LL, JG and QW collected the samples and prepared RNA samples for sequencing. JG, YZ, RS, QW, LL, SR, SL, PL, ZZ, $\mathrm{CL}, \mathrm{BJ}$ analyzed the data. JG and $\mathrm{YZ}$ wrote the manuscript. All authors have read and approved the final manuscript.
Ethics approval and consent to participate

Not applicable.

\section{Consent for publication}

Not applicable.

\section{Competing interests}

The authors declare that they have no competing interests.

\section{Publisher's Note}

Springer Nature remains neutral with regard to jurisdictional claims in published maps and institutional affiliations.

\section{Author details}

${ }^{1}$ Faculty of Information Engineering and Automation, Kunming University of Science and Technology, Kunming 650500, China. ${ }^{2}$ Faculty of Life Science and Technology, Kunming University of Science and Technology, Kunming 650500, China. ${ }^{3}$ Yunnan Key Laboratory of Primate Biomedical Research, Institute of Primate Translational Medicine, Kunming University of Science and Technology, Kunming 650500, China. ${ }^{4}$ Department of Biochemistry and Molecular Biology, Oklahoma State University, Stillwater, Oklahoma 74078, USA.

\section{Received: 30 May 2018 Accepted: 28 November 2018}

Published: 18 April 2019

\section{References}

1. Voinnet O. Origin, biogenesis, and activity of plant MicroRNAs. Cell. 2009;136(4):669-87.

2. Chen X. Small RNAs and their roles in plant development. Annu Rev Cell Dev. 2009;25:21-44.

3. Park W, Li J, Song R, Messing J, Chen X. CARPEL FACTORY, a Dicer homolog, and HEN1, a novel protein, act in microRNA metabolism in Arabidopsis thaliana. Curr Biol. 2002;12(17):1484-95.

4. Bartel DP. MicroRNAs: genomics, biogenesis, mechanism, and function. Cell. 2004;116(2):281-97.

5. Jones-Rhoades MW, Bartel DP, Bartel B. MicroRNAs and their regulatory roles in plants. Annu Rev Plant Biol. 2006;57:19-53. https://doi.org/10. 1146/annurev.arplant.57.032905.105218.

6. Sunkar R, Zhu J-K. Micro RNAs and short-interfering RNAs in plants. J Integr Plant Biol. 2007;49(6):817-26.

7. Pasquinelli AE. MicroRNAs and their targets: recognition, regulation and an emerging reciprocal relationship. Nat Rev Genet. 2012;13(4):271.

8. Sunkar R, Li Y-F, Jagadeeswaran G. Functions of microRNAs in plant stress responses. Trends Plant Sci. 2012;17(4):196-203.

9. Axtell MJ. Classification and comparison of small RNAs from plants. Annu Rev Plant Biol. 2013;64:137-59.

10. Vasudevan $S$, Tong $Y$, Steitz JA. Switching from repression to activation: microRNAs can up-regulate translation. Science. 2007;318(5858):1931-4.

11. Vasudevan S. Posttranscriptional upregulation by microRNAs. Wiley Interdiscip Rev RNA. 2012;3(3):311-30.

12. Xiao M, Li J, Li W, Wang Y, Wu F, Xi Y, Zhang L, Ding C, Luo H, Li Y, et al. MicroRNAs activate gene transcription epigenetically as an enhancer trigger. RNA Biol. 14(10):1326-1334.

13. Liu L, Ren S, Guo J, Wang Q, Zhang X, Liao P, Li S, Sunkar R, Zheng Y Genome-wide identification and comprehensive analysis of micrornas and phased small interfering rnas in watermelon. BMC Genomics. 2018;19(Suppl 2):111.

14. Fei $Q$, Xia R, Meyers BC. Phased, secondary, small interfering RNAs in posttranscriptional regulatory networks. Plant Cell. 2013;25(7):2400-15.

15. Allen E, Xie Z, Gustafson AM, Carrington JC. microRNA-directed phasing during trans-acting siRNA biogenesis in plants. Cell. 2005;121(2):207-21.

16. Xie Z, Allen E, Wilken A, Carrington JC. DICER-LIKE 4 functions in transacting small interfering RNA biogenesis and vegetative phase change in Arabidopsis thaliana. Proc Natl Acad Sci U S A. 2005;102(36):12984-9.

17. Howell MD, Fahlgren N, Chapman EJ, Cumbie JS, Sullivan CM, Givan SA, Kasschau KD, Carrington JC. Genome-Wide Analysis of the RNA-DEPENDENT RNA POLYMERASE6/DICER-LIKE4 Pathway in Arabidopsis Reveals Dependency on miRNA- and tasiRNA-Directed Targeting. Plant Cell. 2007;19(3):926-42. 
18. Johnson C, Kasprzewska A, Tennessen K, Fernandes J, Nan G-L, Walbot V, Sundaresan V, Vance V, Bowman LH. Clusters and superclusters of phased small RNAs in the developing inflorescence of rice. Genome Res. 2009;19(8):1429-40.

19. Song X, Li P, Zhai J, Zhou M, Ma L, Liu B, Jeong D-H, Nakano M, Cao S, Liu C, et al. Roles of DCL4 and DCL3b in rice phased small RNA biogenesis. Plant J. 2012;69(3):462-74.

20. Axtell MJ, Jan C, Rajagopalan R, Bartel DP. A two-hit trigger for siRNA biogenesis in plants. Cell. 2006;127(3):565-77.

21. Chen H-M, Chen L-T, Patel K, Li Y-H, Baulcombe DC, Wu S-H. 22-Nucleotide RNAs trigger secondary siRNA biogenesis in plants. Proc Natl Acad Sci. 2010;107(34):15269-74.

22. Cuperus JT, Carbonell A, Fahlgren N, Garcia-Ruiz H, Burke RT, Takeda A, Sullivan CM, Gilbert SD, Montgomery TA, Carrington JC. Unique functionality of 22-nt miRNAs in triggering RDR6-dependent siRNA biogenesis from target transcripts in Arabidopsis. Nat Struct Mol Biol. 2010;17(8):997-1003.

23. Manavella PA, Koenig D, Weigel D. Plant secondary siRNA production determined by microRNA-duplex structure. Proc Natl Acad Sci. 2012;109(7):2461-6.

24. Xia R, Meyers BC, Liu Z, Beers EP, Ye S, Liu Z. MicroRNA superfamilies descended from miR390 and their roles in secondary small interfering RNA biogenesis in eudicots. Plant Cell. 2013;25(5):1555-72.

25. Rajagopalan $\mathrm{R}$, Vaucheret $\mathrm{H}$, Trejo J, Bartel DP. A diverse and evolutionarily fluid set of microRNAs in Arabidopsis thaliana. Genes Dev. 2006;20(24):3407-25.

26. Chen H-M, Li Y-H, Wu S-H. Bioinformatic prediction and experimental validation of a microRNA-directed tandem trans-acting siRNA cascade in Arabidopsis. PNAS. 2007;104(9):3318-23.

27. Zhai J, Jeong D-H, De Paoli E, Park S, Rosen BD, Li Y, González AJ, Yan Z Kitto SL, Grusak MA, et al. MicroRNAs as master regulators of the plant $N B$-LRR defense gene family via the production of phased, trans-acting siRNAs. Genes Dev. 2011;25(23):2540-53.

28. Shivaprasad PV, Chen H-M, Patel K, Bond DM, Santos BA, Baulcombe DC. A microRNA superfamily regulates nucleotide binding site-leucine-rich repeats and other mRNAs. Plant Cell. 2012;24(3):859-74.

29. Li F, Pignatta D, Bendix C, Brunkard JO, Cohn MM, Tung J, Sun H, Kumar $P$, Baker B. MicroRNA regulation of plant innate immune receptors. Proc Natl Acad Sci. 2012;109(5):1790-5.

30. Källman T, Chen J, Gyllenstrand N, Lagercrantz U. A significant fraction of 21-nucleotide small RNA originates from phased degradation of resistance genes in several perennial species. Plant Physiol. 2013;162(2): $741-54$.

31. Zhu Q-H, Fan L, Liu Y, Xu H, Llewellyn D, Wilson I. miR482 regulation of NBS-LRR defense genes during fungal pathogen infection in cotton. PloS ONE. 2013;8(12):84390.

32. Xia R, Zhu H, An Y-q, Beers EP, Liu Z. Apple miRNAs and tasiRNAs with novel regulatory networks. Genome Biol. 2012;13(6):47.

33. Zhu H, Xia R, Zhao B, An Y-Q, Dardick CD, Callahan AM, Liu Z. Unique expression, processing regulation, and regulatory network of peach (Prunus persica) miRNAs. BMC Plant Biol. 2012;12(1):149.

34. Pei H, Ma N, Chen J, Zheng Y, Tian J, Li J, Zhang S, Fei Z, Gao J. Integrative analysis of miRNA and mRNA profiles in response to ethylene in rose petals during flower opening. PLoS ONE. 2013;8(5):64290.

35. Heinrichs F. International statistics flowers and plants. Hannover: Institut für Gartenbauökonomie der Universität Hannover; 2000.

36. Linde M. Exploring complex ornamental genomes: The rose as a model plant. Crit Rev Plant Sci. 2009;28(4):267-80.

37. Kim J, Park JH, Chan JL, Lim JY, Ryu JY, Lee BW, Choi JP, Kim WB, Lee HY, Choi Y. Small rna and transcriptome deep sequencing proffers insight into floral gene regulation in rosa cultivars. Bmc Genomics. 2012;13(1):657.

38. Grabherr MG, Haas BJ, Yassour M, Levin JZ, Thompson DA, Amit I, Adiconis X, Fan L, Raychowdhury R, Zeng Q. Full-length transcriptome assembly from rna-seq data without a reference genome. Nat Biotechnol. 2011;29(7):644

39. Robinson MD, McCarthy DJ, Smyth GK. edgeR: a Bioconductor package for differential expression analysis of digital gene expression data. Bioinformatics. 2010;26(1):139-40.

40. Xie C, Mao X, Huang J, Ding Y, Wu J, Dong S, Kong L, Gao G, Li C-Y, Wei L. Kobas 2.0: a web server for annotation and identification of enriched pathways and diseases. Nucleic Acids Res. 2011;39(suppl_2):316-22.
41. Zheng Y, Li T, Xu Z, Wai CM, Chen K, Zhang X, Wang S, Ji B, Ming R, Sunkar R. Identification of micrornas, phasirnas and their targets in pineapple. Trop Plant Biol. 2016;9(3):176-86.

42. Burge SW, Daub J, Eberhardt R, Tate J, Barquist L, Nawrocki EP, Eddy SR, Gardner PP, Bateman A. Rfam 11.0: 10 years of RNA families. Nucleic Acids Res. 2013;41(D1):226-32.

43. Bu D, Yu K, Sun S, Xie C, Skogerbø G, Miao R, Xiao H, Liao Q, Luo H, Zhao G, et al. NONCODE v3. 0: integrative annotation of long noncoding RNAs. Nucleic Acids Res. 2012;40(D1):210-5.

44. Quast C, Pruesse E, Yilmaz P, Gerken J, Schweer T, Yarza P, Peplies J, Glöckner FO. The silva ribosomal rna gene database project: improved data processing and web-based tools. Nucleic Acids Res. 2013;41(D1): 590-6. https://doi.org/10.1093/nar/gks1219.

45. Ouyang S, Buell CR. The TIGR Plant Repeat Databases: a collective resource for the identification of repetitive sequences in plants. Nucleic Acids Res. 2004;32(suppl_1):360-3. https://doi.org/10.1093/nar/gkh099.

46. Bao W, Kojima KK, Kohany O. Repbase update, a database of repetitive elements in eukaryotic genomes. Mob DNA. 2015;6(1):1.

47. Li R, Yu C, Li Y, Lam T-W, Yiu S-M, Kristiansen K, Wang J. SOAP2: an improved ultrafast tool for short read alignment. Bioinformatics. 2009;25(15):1966-7.

48. Kozomara A, Griffiths-Jones S. miRBase: annotating high confidence microRNAs using deep sequencing data. Nucleic Acids Res. 2014;42(D1): 68-73.

49. Hofacker IL. Vienna RNA secondary structure server. Nucleic Acids Res. 2003;31(13):3429-31. https://doi.org/10.1093/nar/gkg599.

50. Meyers BC, Axtell MJ, Bartel B, Bartel DP, Baulcombe D, Bowman JL, Cao X, Carrington JC, Chen X, Green PJ, et al. Criteria for annotation of plant MicroRNAs. Plant Cell. 2008;20(12):3186-90.

51. Jagadeeswaran G, Zheng Y, Li Y-F, Shukla LI, Matts J, Hoyt P, Macmil SL, Wiley GB, Roe BA, Zhang W, Sunkar R. Cloning and characterization of small RNAs from Medicago truncatula reveals four novel legume-specific microRNA families. New Phytologist. 2009;184(1):85-98. https://doi.org/ 10.1111/j.1469-8137.2009.02915.x.

52. Zheng Y, Chen K, Xu Z, Liao P, Zhang X, Liu L, Wei K, Liu D, Li Y-F, Sunkar $R$, et al. Small rna profiles from panax notoginseng roots differing in sizes reveal correlation between mir156 abundances and root biomass levels. Sci Rep. 2017;7:9418.

53. Chen K, Liu L, Zhang X, Yuan Y, Ren S, Guo J, Wang Q, Liao P, Li S, Cui X, Li Y-F, Zheng Y. Phased secondary small interfering RNAs in Panax notoginseng. BMC Genomics. 2018;18(S11):10-11861286401743310.

54. De Paoli E, Dorantes-Acosta A, Zhai J, Accerbi M, Jeong D-H, Park S, Meyers BC, Jorgensen RA, Green PJ. Distinct extremely abundant siRNAs associated with cosuppression in petunia. RNA. 2009;15(11):1965-70.

55. Benjamini $Y$, Hochberg $Y$. Controlling the false discovery rate: a practical and powerful approach to multiple testing. J R Stat Soc Ser B Methodol. 1995;57(1):289-300.

56. Zheng Y, Li Y-F, Sunkar R, Zhang W. SeqTar: an effective method for identifying microRNA guided cleavage sites from degradome of polyadenylated transcripts in plants. Nucleic Acids Res. 2012;40(4):28.

57. Zheng Y. Computational Non-codingRNABiology. Cambridge: Academic Press; 2018. 